\title{
I politopi
}

I poliedri sono oggetti belli, interessanti e concreti e hanno attratto le riflessioni dei matematici fin dall'antica Grecia. Sono tra gli oggetti matematici maggiormente studiati e più volentieri considerati dai matematici non professionisti, come dimostrano le centinaia di siti sull'argomento.

A prima vista, i poliedri costituiscono un insieme caotico e poco organizzato di esempi slegati. Ci sono, è vero, i poliedri regolari, quelli archimedei, quelli di Catalan ecc. ma i rapporti tra i diversi poliedri e le loro diverse classificazioni sono spesso molto riposti.

Una delle possibilità per scoprire i loro legami ̀̀, come spesso accade in Matematica, quella di considerarli come casi particolari di oggetti più generali, i politopi, che sarebbero I'analogo dei poliedri in spazi di dimensione maggiore di tre. L'idea di considerare spazi di dimensione maggiore di tre, cosiddetti iperspazi, si è affacciata prepotentemente nel diciannovesimo secolo in diversi campi della Matematica.

L'OHocento è un secolo in cui si assiste a un radicale rinnovamento della Geometria: nascono nuove discipline, come la Geometria proiettiva e le Geometrie non euclidee, e si concepiscono nuovi universi geometrici possibili: quello delle Geometrie non euclidee e quelli degli spazi con dimensione maggiore di 3. Intorno al 1850, Ludwig Schläfli concepisce I'idea di politopo. Schläfli classifica e studia i politopi regolari. In dimensione 3 conosciamo il tetraedro regolare, l'ottaedro, il cubo (esaedro), il dodecaedro e l'icosaedro. In dimensione 4 esistono 6 poliedri regolari e in dimensioni maggiori di quattro ne esistono solo 3 per ogni dimensione.

I politopi possono essere definiti analiticamente assegnando le coordinate dei vertici nello spazio delle $n$-uple di numeri reali, che costituisce il modello analitico dello spazio affine n-dimensionale. Ma i politopi, per poter essere veramente utili, possono e devono anche essere concepiti e intuiti sinteticamente. Spieghiamo brevemente come, partendo da un esempio tridimensionale.

Un cubo si può pensare come generato dinamicamente dalla traslazione di un quadrato (vedi figura 1) lungo la direzione perpendicolare al suo piano.

Analogamente, possiamo pensare che un ipercubo sia generato traslando un cubo nella direzione perpendicolare al suo spazio, cioè lungo una quarta dimensione immaginabile ma non visibile. Il diagramma in figura 2 ci rappresenta la proiezione sul piano del processo di generazione dell'ipercubo, analoga a quella considerata per la generazione del cubo. Per immaginare politopi più complicati, è possibile considerare un processo di generazione più generale. Invece di traslare un poliedro fisso si può immaginare di traslare un poliedro variabile. Come prima, cominciamo da un caso tridimensionale. Immaginiamo di sezionare un cubo con una famiglia di piani paralleli. Possiamo immaginare di ricostruire il cubo incollando le sezioni piane che abbiamo ottenuto precedentemente.

Nel caso di un politopo dello spazio quadridimensionale, non possiamo materialmente sezionare un oggetto che non esiste ma possiamo immaginare tale oggetto come costruito legando insieme una opportuna famiglia di poliedri che costituiscono le sue sezioni iperpiane. Per capire l'idea può essere utile riflettere sulla figura 3 .

Il problema di ricostruire un oggetto dalle sue sezioni è vividamente descritto nel libro di Edwin Abbott, Flatlandia, nel caso tridimensionale e costituisce un esercizio didatticamente molto valido per sviluppare l'intuizione geometrica e la capacità di operare su oggetti tridimensionali. Di ben maggiore difficoltà è il problema di esercitare la propria intuizione geometrica sui politopi partendo dalle loro sezioni iperpiane. Alice Boole fu tra i primi a studiare sistematicamente le sezioni iperpiane di un politopo.

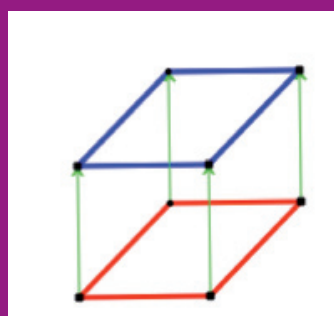

FIGURA 1: UN CUBO SI PUÒ GENERARE TRASLANDO IL QUADRATO ROSSO NELLA DIREZIONE VERTICALE (LA DIREZIONE DEI VETTORI COLORATI IN VERDE). IL QUADRATO ROSSO, NEL SUO MOVIMENTO TRASLATÓRIO PER RAGGIUNGERE IL QUADRATO BLU, GENERA IL CUBO

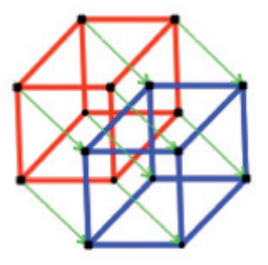

FIGURA 2: L'IPERCUBO VIENE GENERATO TRASLANDO IL CUBO ROSSO NELLA DIREZIONE PERPENDICOLARE AL SUO SPAZIO (LUNGO DEI VETTORI COLORAT IN VERDE). IL CUBO ROSSO, NEL SUO MOVIMENTO TRASLATORIO PER

RAGGIUNGERE IL CUBO BLU, GENERA L'IPERCUBO
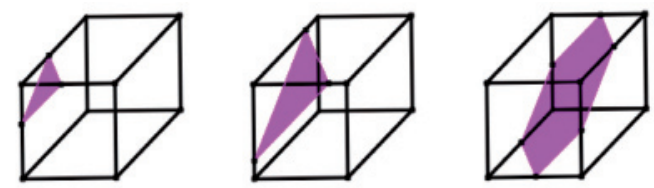

FIGURA 3: IL CUBO VIENE SEZIONATO CON UNA FAMIGLIA DI PIANI PARALLELI. SI NOTI CHE I DIVERSI POLIGONI SEZIONE NON SONO DIVERSI SOLO PER DIMENSIONE, MA ANCHE PER FORMA. SI PASSA DA TRIANGOLI, NELLA PRIMA E SECONDA FIGURA, A ESAGONI NELLA TERZA, PER POI TORNARE A TRIANGOLI CONTINUANDO A SPOSTARE IL PIANO CON CUI SI OPERA LA SEZIONE PARALLELAMENTE A SE STESSO
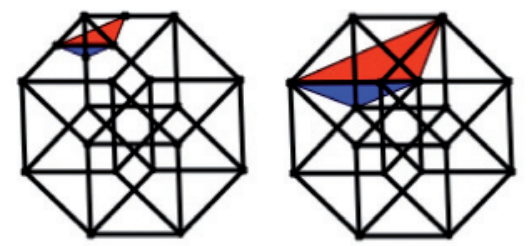

FIGURA 4: L'IPERCUBO VIENE SEZIONATO CON UNA FAMIGLIA DI IPERPIANI PARALLELI. SONO MOSTRATE LE PROIEZIONI PIANE DI DUE SEZIONI CHE SONO TETRAEDRI REGOLARI. SEZIONANDO CON ULTERIORI IPERPIANI SI OTTENGONO OTTAEDRI E POI ANCORA TETRAEDRI. ALICIA BOOLE DETERMINÒ LE SEZIONI IPERPIANE ORTOGONALI AD ALCUNE DIREZIONI PRIVILEGIATE DI TUTTI I POLITOPI REGOLARI QUADRIDIMENSIONALI 